\title{
Essential Role of Interferon Response in Containing Human Pathogenic Bourbon Virus
}

\author{
Jonas Fuchs, Tobias Straub, Maximilian Seidl, Georg Kochs
}

Bourbon virus (BRBV) is a recently discovered tick-transmitted viral pathogen that is prevalent in the Midwest and southern United States. Since 2014, zoonotic BRBV infections have been verified in several human cases of severe febrile illness, occasionally with fatal outcomes, indicating a possible public health threat. We analyzed the pathology of BRBV infection in mice and found a high sensitivity of the virus to the host interferon system. Infected standard laboratory mice did not show clinical signs or virus replication. However, in mice carrying defects in the type I and type II interferon system, the virus grew to high titers and caused severe pathology. In cell culture, BRBV was blocked by antiviral agents like ribavirin and favipiravir (T705). Our data suggest that persons having severe BRBV infection might have a deficiency in their innate immunity and could benefit from an already approved antiviral treatment.

$\mathrm{I}$ $\mathrm{n}$ the past 4 years, several reports from the Midwest and southern United States have described the detection of a new viral pathogen, called Bourbon virus (BRBV), associated with severe febrile illness (1-4). The isolation of BRBV from patients with a history of tick bites was unexpected, because BRBV belongs to tick-transmitted viruses of the genus Thogotoviruses, which are largely unknown in the United States. However, recent tick surveillance campaigns confirmed the prevalence of BRBV in the affected region $(5,6)$.

Thogotoviruses are influenza virus-like arboviruses with a segmented RNA genome; they are frequently isolated in Africa, the Middle East, Asia, and southern Europe and are usually associated with diseases in livestock (7). BRBV is genetically most similar to Dhori virus (DHOV) from India (8). Although serologic surveys suggest the occurrence of zoonotic transmission of Thogotoviruses, few human cases have been well-documented $(9,10)$. However, in laboratory mice, Thogotoviruses show an aggressive systemic infection affecting mainly

Author affiliation: University of Freiburg Medical Center, Faculty of Medicine, University of Freiburg, Freiburg, Germany

DOI: https://doi.org/10.3201/eid2507.181062 the liver, lungs, and spleen, leading to a fatal acute hepatitis. This severe disease progression is accompanied by a massive induction of interferon (IFN) $\alpha$ without an apparent protective effect $(11,12)$.

We conducted our study with the aim to evaluate the virulence and pathogenesis of BRBV in vivo. Furthermore, we assessed the antiviral effect of the host IFN system on BRBV replication.

\section{Materials and Methods}

\section{Biosafety and Animal Ethics}

Because of the unknown health risk associated with the human BRBV isolate, all work with infectious virus was performed under Biosafety Level 3 conditions. The animals were handled in accordance with guidelines of the Federation for Laboratory Animal Science Association and the national animal welfare body of Germany. Animal experiments were performed in compliance with animal protection laws in Germany and approved by the local animal welfare committee (Regierungspraesidium Freiburg, permit no. G-15/127).

\section{Cells and Viruses}

We cultivated human lung epithelial A549 cells (ATCC CCL-185), human hepatoma Huh7 cells (13), human cervix carcinoma HeLa cells (ATCC CCL-2), African green monkey kidney Vero cells (ATCC CCL-81), and transformed human dermal fibroblast cultures (14) in Dulbecco modified eagle medium supplemented with $10 \%$ fetal calf serum at $37^{\circ} \mathrm{C}$ and $5 \% \mathrm{CO}_{2}$. We treated Huh7 cells with recombinant human IFN- $\alpha 2$ a (PBL Assay Science, https://www.pblassaysci.com) or recombinant human IFN- $\gamma$ (R\&D Systems, https://www.rndsystems.com) 16 $\mathrm{h}$ before and $2 \mathrm{~h}$ after infection. To test antivirals, we treated Huh7 cells with ribavirin (Sigma-Aldrich, https:// www.sigmaaldrich.com) or favipiravir/T-705 (BioVision, https://www.biovision.com) $2 \mathrm{~h}$ after infection. To evaluate the cytotoxicity of these substances, we treated uninfected cells for $48 \mathrm{~h}$ with the maximum doses as 
described. Afterward, we assessed the viability of the cells by measuring the activity of lactate dehydrogenase in the cell culture supernatant (Pierce LDH Cytotoxicity Assay Kit; ThermoFisher, https://www.thermofisher. com). To establish a positive control, we treated cells with the lysis buffer provided by the manufacturer.

\section{Viruses and Infection}

BRBV (strain NR-50132/ATCC VR-1842) (1) was kindly provided by Amy J. Lambert of the Centers for Disease Control and Prevention (Fort Collins, CO, USA). We produced virus stocks of BRBV, DHOV (strain India/1313/61) (15), and Thogoto virus (THOV) (strain SiAr126) (16) on Vero cells. To perform growth kinetics, we infected the cells with BRBV at a multiplicity of infection (MOI) of 0.001 in Dulbecco modified eagle medium with $1 \%$ fetal calf serum and $20 \mathrm{mmol} / \mathrm{LM}$ HEPES. We harvested supernatants at the indicated time points. We stored viruscontaining supernatants at $-80^{\circ} \mathrm{C}$, and we determined viral titers by using plaque assay on Vero cells.

\section{Animal Infections}

We purchased wild-type C57BL/6 mice from Janvier Labs (https://www.janvier-labs.com). We bred mice with defects in the IFN pathway (17) in house. We conducted all experiments by using sex- and age-matched animals (7-9 week-old mice). We infected animals intraperitoneally with BRBV or DHOV diluted in $100 \mu \mathrm{L}$ PBS with $0.3 \%$ bovine serum albumin. Depending on the experimental setup, we measured weight, survival, and clinical score daily for each animal (Appendix, https://wwwnc.cdc.gov/ EID/article/25/7/18-1062-App1.pdf). We euthanized the animals by using cervical dislocation at the indicated time points. To determine survival of the animals after infection, we euthanized animals if the weight loss was $>25 \%$ or the mice showed severe clinical signs. We harvested organs (liver, lung, spleen, and kidney) at day 4 postinfection and homogenized them by using FastPrep Homogenizer (MP Biomedicals, https://www.mpbio.com) in PBS. After centrifugation of the supernatants at $5,000 \times g$ for 10 min at $4^{\circ} \mathrm{C}$, we analyzed them by using plaque assay on Vero cells. We collected whole blood from animals anesthetized with ketamine/xylazine by using heart puncture before cervical dislocation. We prepared serum samples by using incubation at $37^{\circ} \mathrm{C}$ for $10 \mathrm{~min}$ and centrifugation at $5,000 \times g$ for $10 \mathrm{~min}$. We used serum samples directly to determine alanine transaminases by using an alanine color endpoint assay (MaxDiscovery; Bio Scientific, http://www. biooscientific.com), or we stored the samples at $-20^{\circ} \mathrm{C}$. We generated postinfectious serum directed against BRBV by challenging C57BL/6 mice with $10^{5}$ PFU/animal. Fourteen days after infection, we harvested the serum. Because of the lethality of DHOV, we used $\mathrm{Mx}^{+/+}$mice for the infection and production of specific antiserum directed against DHOV, as described previously (18).

We performed antibody treatment of the animals by intraperitoneal injection. To deplete IFN- $\gamma$, we injected $0.5 \mathrm{mg}$ of IFN- $\gamma$ monoclonal antibody (mAb) (XMG1.2; Biolegend, https://www.biolegend.com) at 1 day preinfection and 2 days postinfection. We achieved blockage of the type I IFN receptor (IFNAR) by treating the mice with $1 \mathrm{mg}$ of anti-IFNAR-1 mAb (MAR1-5A3; BioXCell, https://bxcell.com) at 1 day preinfection and 1 day postinfection. To deplete natural killer (NK) cells, we treated IFNAR $^{-/}$mice infected with 100 PFU of BRBV with 0.25 $\mathrm{mg}$ of NK1.1 mAb (PK136, BioXcell) at 3 days preinfection and 1 day postinfection. At 4 days postinfection, we harvested organs and used fluorescence-activated cell sorting analysis to determine virus titers and confirm the depletion of NK $1.1^{+}$cells.

We administered $20 \mathrm{mg}$ or $40 \mathrm{mg}$ of ribavirin $(5 \mathrm{mg} /$ $\mathrm{mL}$ in $0.9 \% \mathrm{NaCl}$; Sigma-Aldrich, https://www.sigmaaldrich.com) per kilogram bodyweight each day intraperitoneally, starting immediately postinfection. We mock-treated control animals with $0.9 \% \mathrm{NaCl}$ only.

For histologic analysis, we harvested the organs, which we washed once in PBS and then fixed in 4\% formaldehyde in PBS overnight. For cryoprotection, we incubated organs once in $15 \%$ sucrose (in $\mathrm{H}_{2} \mathrm{O}$ ) at $4^{\circ} \mathrm{C}$ for $4 \mathrm{~h}$ and afterward in $30 \%$ sucrose at $4{ }^{\circ} \mathrm{C}$ overnight. After embedding in OCT medium (Tissue-Tek; Sakura, https://www.sakuraus.com), we performed 5 - $\mu \mathrm{m}$ cryosections and stained them with hematoxylin and eosin.

\section{Western Blot Analysis and Antibodies}

We infected Vero cells with the indicated viruses (MOI 0.25) for $24 \mathrm{~h}$ and then lysed them in Passive Lysis Buffer (Promega, https://www.promega.com). We denaturated proteins in Lämmli buffer and incubated them at $95^{\circ} \mathrm{C}$ for $5 \mathrm{~min}$. We separated the protein lysates by using $12 \%$ SDS-polyacrylamid gel electrophoresis and transferred them onto a PVDF membrane (Millipore Sigma, http://www.emdmillipore.com). We detected viral proteins by using polyclonal mouse antisera. We used $\beta$-actinspecific rabbit antiserum (Sigma-Aldrich) as an internal control. We detected primary antibodies by using fluorescent-labeled anti-mouse secondary antibodies (LI-COR, https://www.licor.com).

To test the antiserum for virus neutralization, we prepared serial dilutions of the polyclonal mouse serum in PBS and incubated them with a fixed amount of $100 \mathrm{PFU}$ of BRBV for $1 \mathrm{~h}$ at room temperature. To establish a control, we incubated virus with PBS or an unspecific mouse serum. We transferred the virus-serum mixture onto Vero cells and performed a plaque assay. We normalized the PFU of the antibody-treated viruses to the control virus. 


\section{Real-Time Reverse Transcription PCR}

RNA was extracted (NucleoSpin RNA kit; MachereyNagel, https://www.mn-net.com) from infected cells and subjected to cDNA synthesis (QuantiTect Reverse Transcription Kit; QIAGEN, https://www.qiagen.com). We performed real-time reverse transcription PCR (RT-PCR) by using $10 \mathrm{ng}$ cDNA in a SYBR Green assay (QuantiTect PCR Kit, QIAGEN) with primers specific for human IFN- $\beta$ (Hs_IFNB1_1, QIAGEN) and human $\gamma$-actin (Hs_ACTG1_1, QIAGEN). We normalized cycle threshold values to actin $(\triangle \mathrm{CT})$ and plotted them relative to the $\Delta \mathrm{CT}$ values of the mock-treated control $\left(2^{-\Delta \Delta \mathrm{CT}}\right)$. We detected viral transcripts of BRBV and DHOV by using panspecific Thogotovirus primers (FW: TTCAATGAATGYTTGGACCCAGATGC [segment 2, nucleotides 940-965]; RW: TTGWACATYCCCATGAACAT [segment 2, nucleotides $1,210-1,229])$ in a conventional RT-PCR; we detected the products by using an ethidium bromide-stained agarose gel.

\section{Statistical Analyses}

We analyzed all data with Prism 7 software (GraphPad, https://www.graphpad.com). We performed statistical analysis of viral titers on log-transformed values by using a 1-way analysis of variance with a Tukey multiple comparison test (for $\geq 3$ groups) or a 2 -sided $t$-test (for 2 groups). We plotted viral titers either on a log scale (geometric mean) or log transformed on a linear scale (mean $\pm \mathrm{SD}$ ). For weight loss, we calculated SEM.

\section{Results}

\section{BRBV Sensitivity to Type I and Type II IFN}

We studied the virulence of BRBV by infection of C57BL/6 (B6) mice with high challenge doses of BRBV that did not result in disease (Figure 1, panel A), as reported previously (8). The lack of pathogenicity is surprising because related Thogotoviruses regularly cause generalized
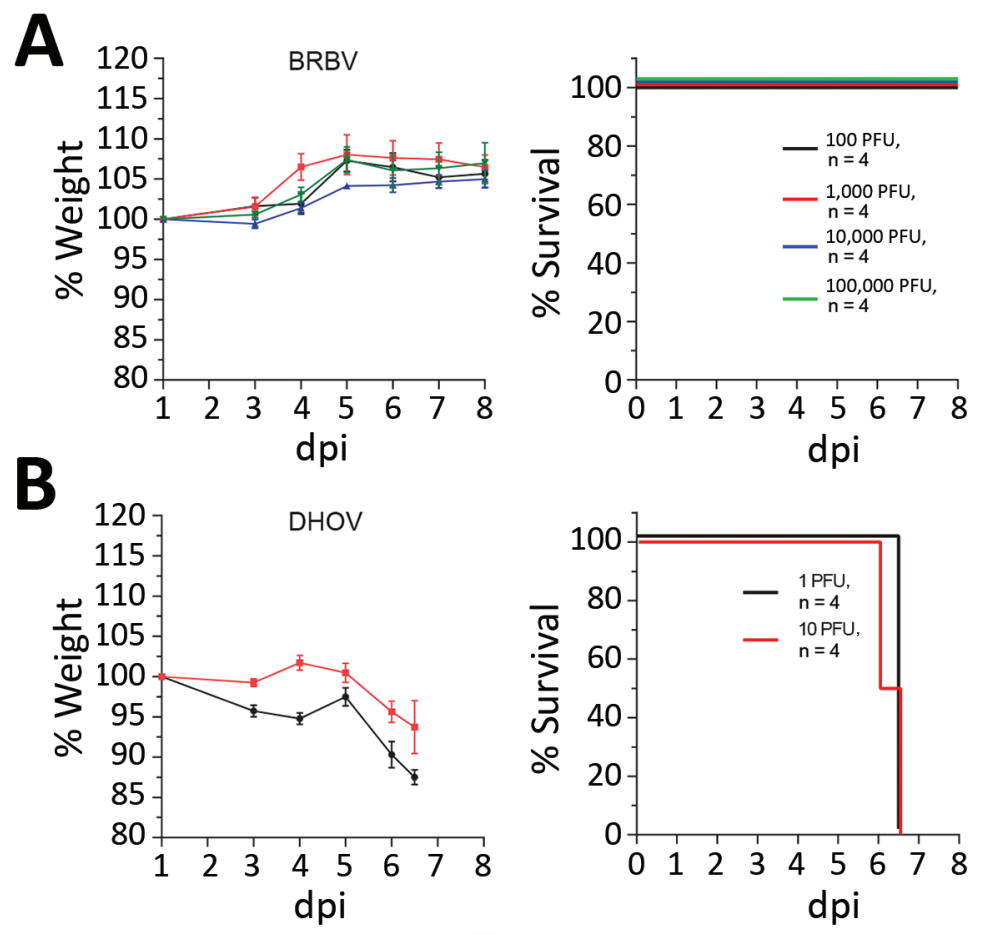

D
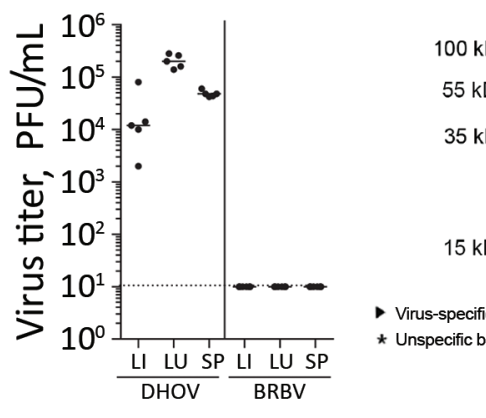

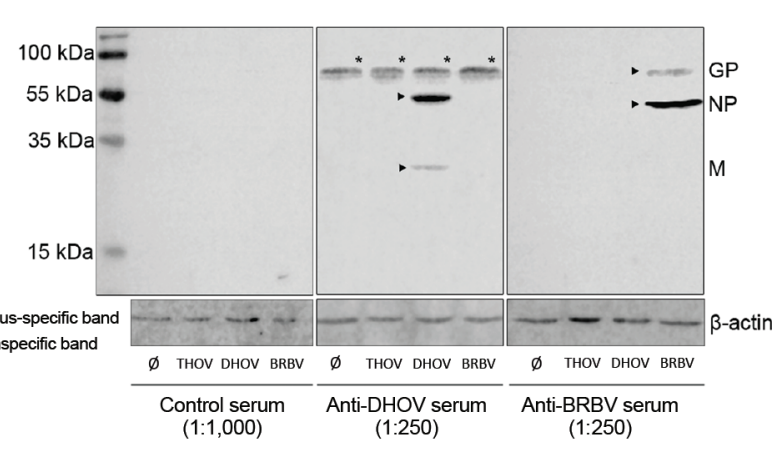

Figure 1. Results of virulence testing of BRBV in immunocompetent mice. A, B) B6 mice $(n=4)$ infected intraperitoneally with the indicated doses of BRBV $(A)$ or DHOV (B) were monitored for weight (mean \pm SEM) and survival. Animals were euthanized if they lost $>25 \%$ body weight or showed severe illness. C) For virus growth, B6 mice $(n=5)$ were infected intraperitoneally with 100 PFU of DHOV and 1,000 PFU of BRBV. After 4 days, liver, lung, and spleen were harvested and a plaque assay performed. Geometric means are shown; dotted line indicates detection limit. D) Serum samples from BRBV- or DHOV-infected mice were pooled at $14 \mathrm{dpi}$. Vero cells were mock-treated or infected (multiplicity of infection 0.25 ) with BRBV, DHOV, and THOV and Western blot analysis of lysates from infected cells performed with antiserum at the indicated dilutions. Molecular weight and identity of the viral antigens are indicated. BRBV, Bourbon virus; DHOV, Dhori virus; dpi, days postinfection; GP, glycoprotein; LI, liver; LU, lung; $M$, matrix protein; NP, nucleoprotein; SP, spleen; THOV, Thogotovirus; $\varnothing$, mock-treated (control). 
severe infections in IFN-competent laboratory mice (11). Infection of B6 mice with the closely related DHOV led to severe illness and early death (Figure 1, panel B). Virus replication was detectable in liver, lung, and spleen of DHOV-infected but not BRBV-infected animals (Figure 1, panel C). Successful BRBV infection was confirmed by seroconversion (Figure 1, panel D) and generation of neutralizing antibodies (Appendix Figure).

BRBV replicated to high titers in cells that are defective in IFN responses such as simian Vero or human Huh7 cells $(13,19)$, as previously reported (8) (Figure 2, panel A). In IFN-responsive human A549 and HeLa cells, virus growth was reduced by $2 \operatorname{logs}$ (Figure 2, panel A). Realtime RT-PCR analysis showed that BRBV and DHOV strongly induced IFN- $\beta$ expression in A549 cells, whereas Huh7 cells were unresponsive, as reported previously (13). Both cell lines were efficiently infected, as demonstrated by viral PB1 gene expression (Figure 2, panel B). To confirm the role of type I IFN in the suppression of BRBV, we infected A549 cells that were stably overexpressing the IFNantagonistic Npro protein of bovine viral diarrhea virus or the $\mathrm{V}$ protein of simian virus 5 . Npro inhibits IFN synthesis by targeting transcription factor IRF3 (20), and the V protein blocks IFN signaling by targeting STAT1 for degradation (21). Both cultures showed enhanced BRBV replication compared with the parental A549 control cells (Figure 2, panel C), indicating that BRBV-induced IFN elicited an antiviral state that suppressed virus propagation. Enhanced growth of BRBV was also observed in transformed dermal fibroblast cultures obtained from an IFN-nonresponsive person with a genetic defect in STAT2 (14) (Figure 2, panel D). BRBV clearly is highly sensitive to the antiviral state induced by IFN.

We obtained similar results in vivo using IFN-nonresponsive animals. Mice lacking IFN- $\alpha / \beta$ receptor expression $\left(\mathrm{IFNAR}^{-/}\right.$) cannot respond to type I IFN, whereas

Figure 2. Results of sensitivity testing of BRBV to type I IFN-induced antiviral state in cell culture. A) Vero, Huh7, A549, and HeLa cells were infected with BRBV (multiplicity of infection [MOI] 0.001). At the indicated time points, the supernatants were harvested and viral titers determined. B)

Huh7 or A549 was infected (MOI

0.25 ) with BRBV or DHOV for

$16 \mathrm{~h}$. Whole RNA was extracted and IFN- $\beta$ and actin transcripts detected by real-time reverse transcription PCR. Changes in IFN- $\beta$ transcripts were calculated in comparison to mock-treated cells. A conventional reverse transcription PCR assay with panspecific primers for viral segment 2 (PB1) was performed to control the infection. $C, D$ ) Defects in IFN induction or signaling enhancing BRBV propagation. Parental A549 and A549 cells stably expressing Npro of bovine viral diarrhea virus or the $\mathrm{V}$ protein of simian virus $5(C)$, as well as (D) human skin fibroblast control cultures or with a defect in STAT2 infected with BRBV (MOI 0.001). Culture supernatants were collected and viral titers determined. Shown are the arithmetic means $(\underline{ \pm} \mathrm{SD})$ of log-transformed values of 3 independent experiments. Statistical analyses were performed with a 1-way analysis of variance (Tukey multiple comparison test) (C) or a 2-tailed $t$-test (D). BRBV, Bourbon virus; CTRL, control; DHOV, Dhori virus; IFN, interferon;

NS, nonsignificant; $\varnothing$, mocktreated (control). ${ }^{* * *} p<0.001$; ${ }^{* *} p<0.01 ;{ }^{*} p<0.05$.
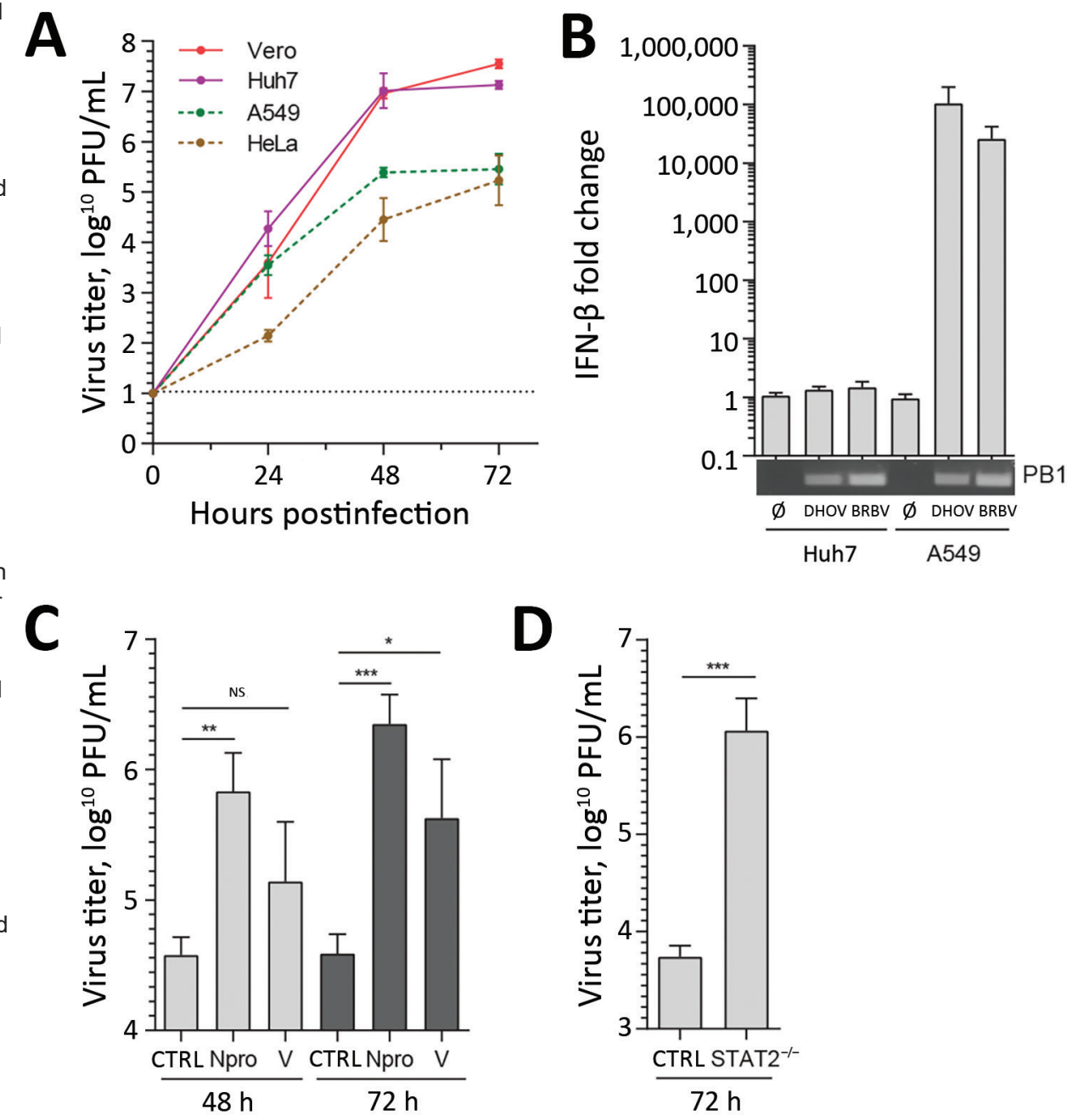
mice devoid of both IFN- $\alpha / \beta$ and IFN- $\lambda$ receptor expres-

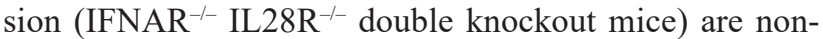
responsive to type I and type III IFNs (17). Although both types of mice supported BRBV growth in liver, lung, spleen, and kidney (Figure 3, panel A), virus growth was not detectable in single IL28R $\mathrm{R}^{-1-}$ mice, indicating that type III IFN plays a minor role. Unexpectedly, STAT1 $1^{-/}$mice were even more susceptible than the IFN receptor-deficient animals, and BRBV grew to higher titers (Figure 3 , panel A). We reasoned that IFN- $\gamma$ (type II IFN) might have a role in controlling virus replication because, like IFN- $\alpha / \beta$, IFN- $\gamma$ signaling relies on STAT1 for signal transduction and antiviral activity. We therefore treated B6 mice with monoclonal antibodies directed either against IFN- $\gamma$ or against IFNAR. Blocking IFN- $\gamma$ had no effect, whereas blocking IFNAR led to detectable BRBV replication in most organs tested except the liver (Figure 3, panel B).Treatment with a mixture of both antibodies massively increased virus growth in all organs, including the liver (Figure 3, panel B), indicating that type I and type II IFNs worked synergistically. The synergistic effect was also reflected in the severity of disease.

Treatment with IFNAR but not IFN- $\gamma$ antibodies led to a transient drop in body weight with rapid recovery (Figure 3 , panel C). In contrast, bodyweight loss in mice treated with both antibodies was dramatic (Figure 3, panel C), and the animals had to be euthanized 4 days after infection (Figure 3, panel D). The same effect was observed in IF$\mathrm{NAR}^{-/-}$IFNLR $^{-/}$double knockout mice treated with IFN- $\gamma$ antibody (Figure 3, panels E-G). IFN- $\alpha / \beta$ clearly was the dominant antiviral factor controlling BRBV, but its antiviral effect was potentiated by IFN- $\gamma$, which is known to act on myeloid cells (22), the main target cells of Thogotoviruses (23). NK cells are known as the major producers of IFN- $\gamma$ early during acute infections (24). However, depletion of NK cells by treatment of IFNAR ${ }^{--}$mice with NK1.1 antibody could not elevate virus replication (data not shown), leaving the identity of the IFN- $\gamma$-producing cell compartment unclear.

We investigated the clinical and pathologic changes in type I (IFNAR ${ }^{--}$) and type I and II (STAT1 ${ }^{-/-}$) IFN-nonresponsive mice after BRBV infection (Figure 4). Wild-type mice remained asymptomatic throughout the course of infection and showed no gross abnormalities. STAT $1^{-/-}$mice showed the most severe clinical score and became moribund, whereas IFNAR ${ }^{-/}$mice were less severely affected and survived (Figure 4, panel A). Serum alanine aminotransferase levels were elevated as a sign of liver damage (Figure 4, panel B). Histologic liver sections showed massive hepatocellular destructions, including pathologic lesions and infiltrating inflammatory cells (Figure 4, panel C), consistent with acute degenerative hepatitis. Furthermore, the spleens of STAT1 ${ }^{--}$animals showed massive necrosis and destruction of the normal architecture (Figure 4, panel C). We also detected infiltrations of granulocytes in lungs and kidneys of the infected STAT $1^{-/}$animals, indicating mild inflammation without apparent pathologic changes (data not shown).

The pathologic and clinical signs observed in mice were in some aspects compatible with the clinical manifestations described for the severe human BRBV cases in 2014 and $2017(1,3)$. The mice showed a high degree of liver damage and elevated liver enzymes, as did the human patients. At later stages, the human patients had acute respiratory complications. Accordingly, the infected STAT1 $1^{-/}$mice showed high virus replication and infiltration of lymphocytes in their lungs accompanied by labored breathing. However, the prominent maculopapular rash that was described in both human patients was not observed in the STAT $1^{-/}$mice.

\section{Antiviral Treatment Effect of Blocking BRBV Replication and Diminishing Pathology}

As stated recently by the Centers for Disease Control and Prevention, no antiviral treatment of BRBV disease is available (25). Therefore, we tested the protective effect of IFN- $\alpha$ and IFN- $\gamma$ in Huh7 cells. Treatment with IFN- $\alpha$ led to a 100 -fold reduction in viral titers, whereas treatment with IFN- $\gamma$ was less efficient. Combination of both cytokines led to $>1,000$-fold reduced virus titers, consistent with a synergistic mode of action (Figure 5, panel A). Treatment with the guanosine analog (ribavirin) and the guanine analog (favipiravir [T705]), both known inhibitors of viral RNA-dependent RNA polymerases $(26,27)$, resulted in an up to 1 million-fold titer reduction (Figure 5, panels B, C). To evaluate the effect of ribavirin in combination with increasing amounts of IFN- $\alpha$, we used moderate concentrations that correspond to pharmacologically relevant serum concentrations of 4-8 $\mu \mathrm{mol} / \mathrm{L}$ for ribavirin and $100 \mathrm{IU} / \mathrm{mL}$ for IFN $\alpha(28)$ and observed an up to 1,000-fold reduction of virus replication (Figure 5, panel D). We did not observe cytotoxicity of IFN- $\alpha$, IFN- $\gamma$, ribavirin, or favipiravir (T705) (Figure 5, panel E).

Our mouse models might be suitable to test new treatment regimens to curtail BRBV infections. IFNAR ${ }^{-/}$and $\mathrm{STAT}^{-/}$mice were infected with a high dose of BRBV and treated daily with ribavirin. The amount of ribavirin used in these experiments was similar to that used in published experiments on influenza virus infections in mice (26) and the range of ribavirin treatment of hepatitis $\mathrm{C}$ patients (28). In our study, treatment reduced BRBV replication in most organs and resulted in efficient titer reductions in liver, lung, and kidney (Figure 5, panel F). Treatment also resulted in reduced weight loss (Figure 5, panel G) and a significant delay of 5 days in the mean time until death 

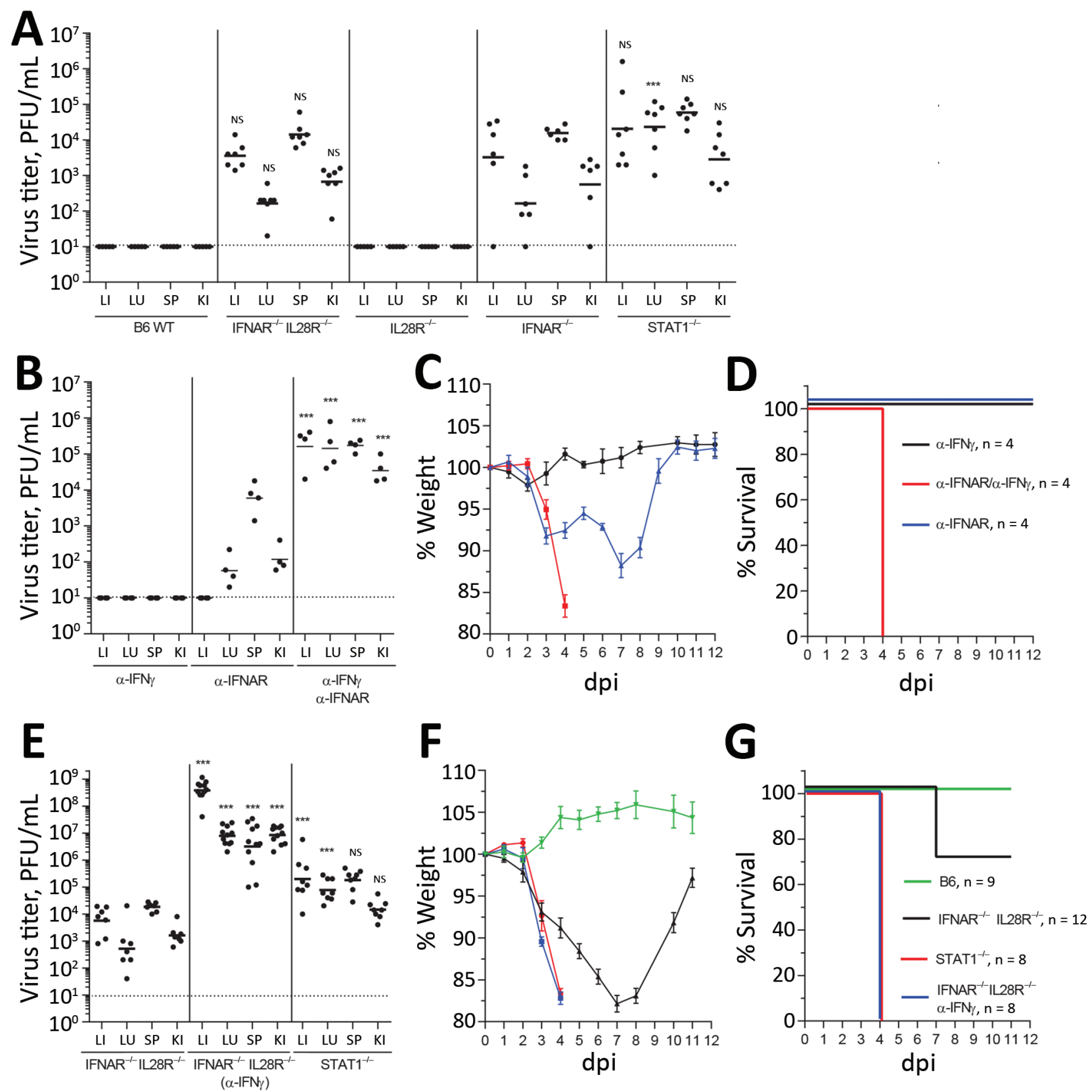

Figure 3. Immune response to BRBV in vivo. A) B6 WT $(n=5)$ mice or animals with a knockout in IFNAR $\left(\right.$ IFNAR $\left.{ }^{---}\right)(n=6)$, IL28R ${ }^{-1-}$ $(n=6)$, IFNAR ${ }^{-1-}$ IL28R $^{--}$double knockout $(n=6)$, or STAT1 ${ }^{-1-}(n=7)$ infected intraperitoneally with BRBV $(1000$ pfu). Liver, lung, spleen, and kidney were harvested at day 4 and viral titers determined. B) B6 WT animals $(n=4 / g r o u p)$ treated with monoclonal antibodies directed against IFNAR-1 $(0.5 \mathrm{mg} / \mathrm{mouse} 24 \mathrm{~h}$ before and $24 \mathrm{~h}$ after infection) or against IFN- $\gamma(1 \mathrm{mg} / \mathrm{mouse} 24 \mathrm{~h}$ before and $48 \mathrm{~h}$ after infection) and infected with BRBV (100 PFU) for $4 \mathrm{~d}$. C, D) B6 WT animals (4 per group) treated as in panel B and weight loss (mean \pm SEM) and survival monitored. Animals were euthanized if they lost $>25 \%$ body weight or showed severe clinical signs. $E$ ) IFNAR $^{--}$IL28R $^{-1-}$ mice treated with $\alpha-\operatorname{IFN} \gamma$ antibody $(n=11)$ or left untreated $(n=7)$ as described in panels B-D and STAT1 $1^{--}(n=8)$ animals infected with BRBV (100 PFU). At day 4, postinfection viral titers were determined. $F, G)$ The mice (E) together with additional B6 WT $(n=9)$ were monitored for weight loss (mean \pm SEM) and survival as in panels $C$ and $D$. In panels $A, B$, and $E$, geometric means are displayed and dotted lines indicate detection limits. Statistical analyses were performed on log-transformed values with a 1-way analysis of variance (Tukey multiple comparison test). Statistics are presented in comparison to the respective organs of IFNAR ${ }^{-1-}(A)$, a-IFNAR (B), or IFNAR ${ }^{-1-}$ IL28R ${ }^{-1-}(E)$. BRBV, Bourbon virus; dpi, days postinfection; IFN, interferon; IFNAR, type I interferon receptor; KI, kidney; LI, liver; LU, lung; NS, nonsignificant; SP, spleen; WT, wild-type. ${ }^{* * *} p<0.001$. 
in the BRBV-infected STAT1 ${ }^{-1}$ mice (Figure 5, panel H). Our results suggest that ribavirin, possibly in combination with pegylated IFN- $\alpha$, might be an antiviral treatment option, as in the case of hepatitis C (29).

\section{Discussion}

Characterization of the human BRBV isolate demonstrated a surprisingly strong sensitivity to the type I and type II IFN system. Our results indicate that the few persons
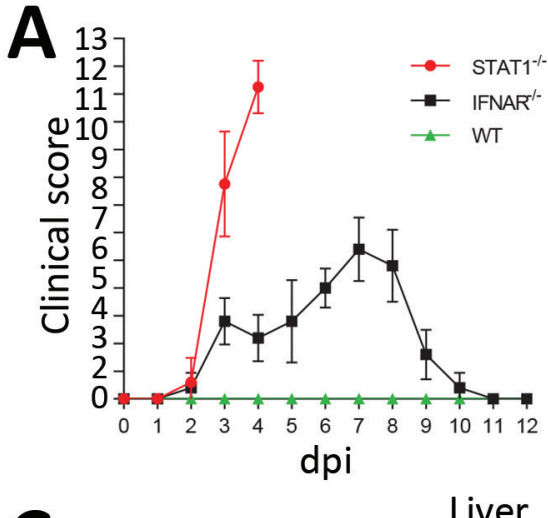

C
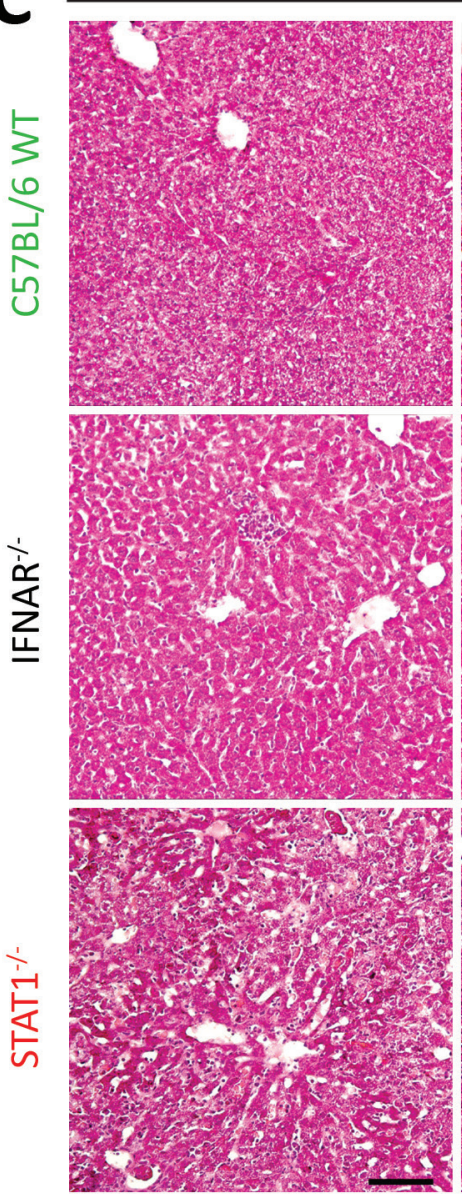

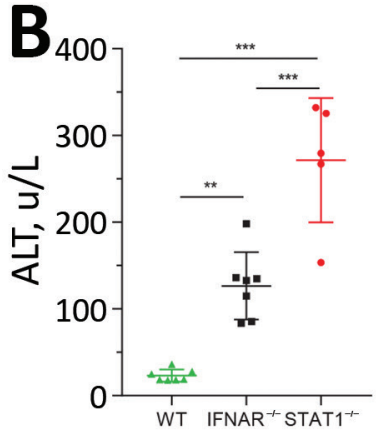

WT IFNAR ${ }^{+}$STAT $^{-1}$
Liver
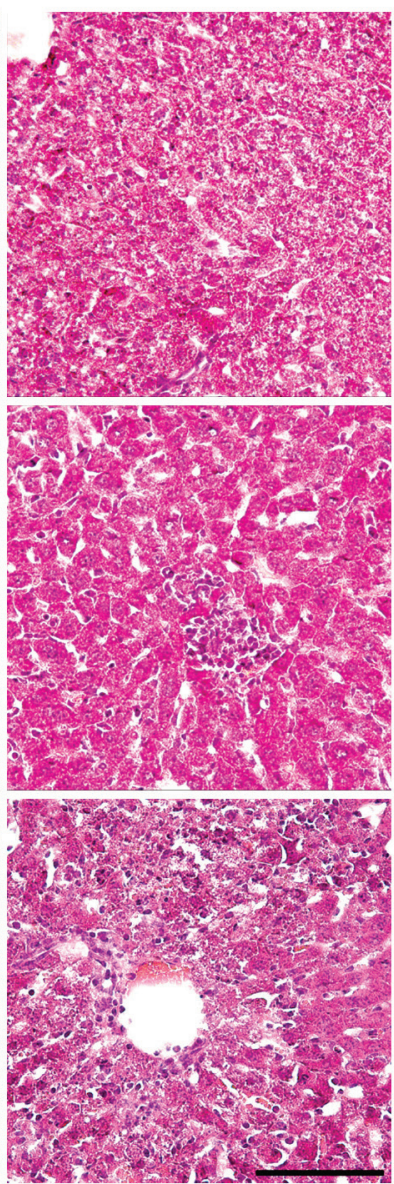

Spleen
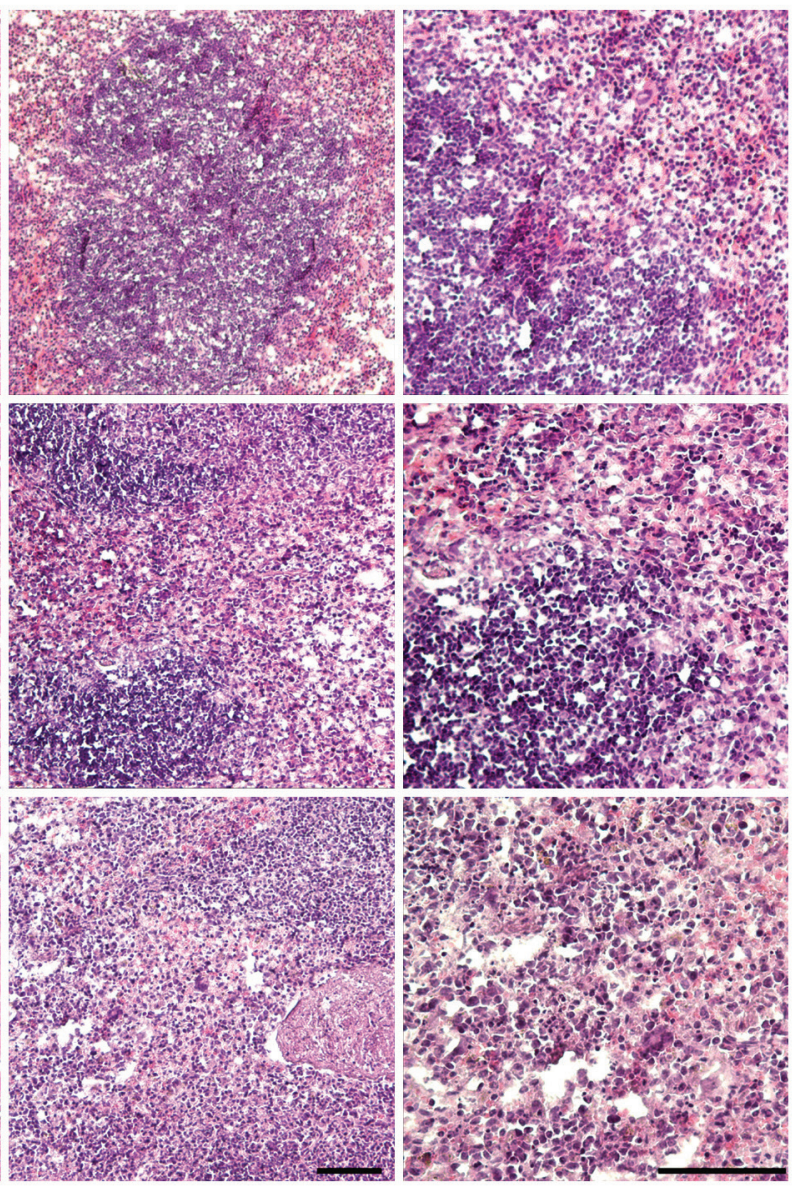

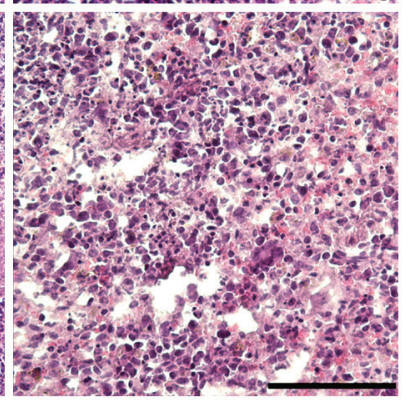

Figure 4. Pathology of BRBV-infected mice. A) B6 WT, IFNAR ${ }^{-1-}$, and STAT1 ${ }^{-1-}$ animals $(n=5$ for each group) infected intraperitoneally with 100 PFU of BRBV and monitored daily for weight and clinical signs (mean +SD); scoring system described in Appendix Table (https://wwwnc.cdc.gov/EID/article/25/7/18-1062-App1.pdf). B) Serum samples of infected B6 WT $(n=7)$, IFNAR ${ }^{--}(n=7)$, and STAT1 ${ }^{-1}$ $(n=5)$ animals harvested at day 4 and analyzed for ALT (mean +SD). Statistical analysis was performed with a 1-way analysis of variance (Tukey multiple comparison test). C) Histologic results showing inflammatory organ damage at 4 dpi. Organs were fixed in $4 \%$ formaldehyde and embedded in OCT medium (Tissue-Tek; Sakura, https://www.sakuraus.com); cryosections were then stained with hematoxylin and eosin. Scale bars indicate $100 \mu \mathrm{m}$. ALT, alanine aminotransferase; BRBV, Bourbon virus; dpi, days postinfection; IFNAR, type I interferon receptor; WT, wild-type. ${ }^{* *} p<0.001 ;{ }^{* *} p<0.01$. 
to date who had a severe BRBV infection might have had an inborne or transient weakness in their innate antiviral immune response. Also, in case of an acute symptomatic infection with BRBV, our study provides a potential therapeutic option based on the long-approved treatment with ribavirin, possibly in combination with IFN- $\alpha$.

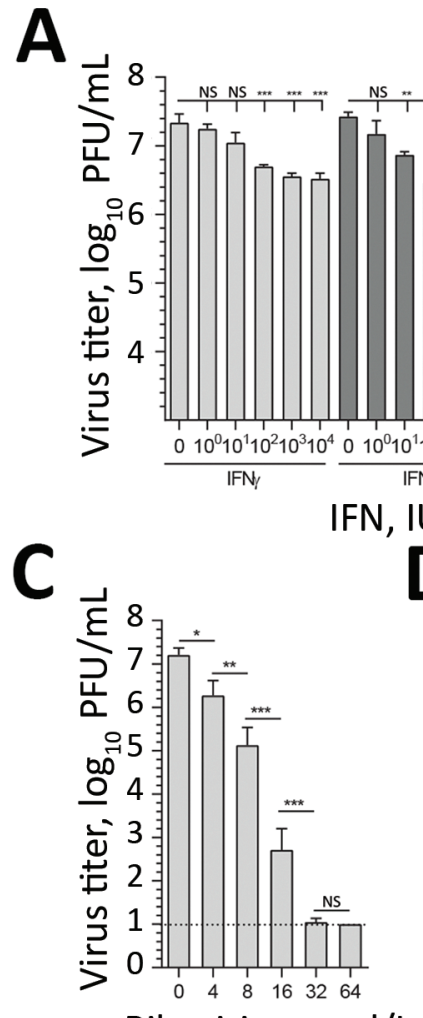

Ribavirin, $\mu \mathrm{mol} / \mathrm{L}$

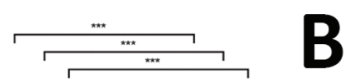

N NS ...

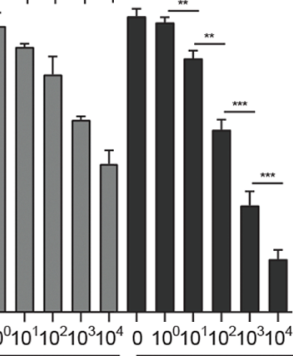

IFNa2a $\frac{\text { IFNa2a+IFNy }}{\text { IF }}$

IFNk2a
$F$

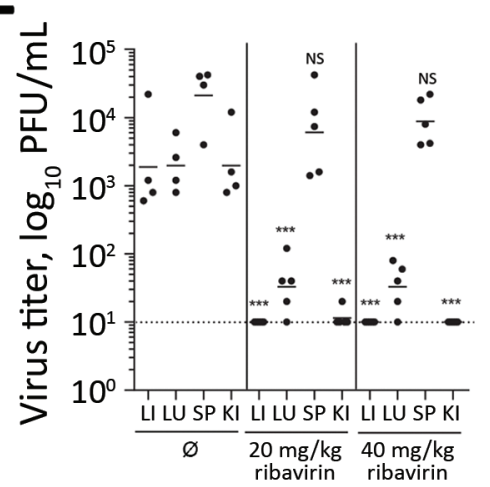

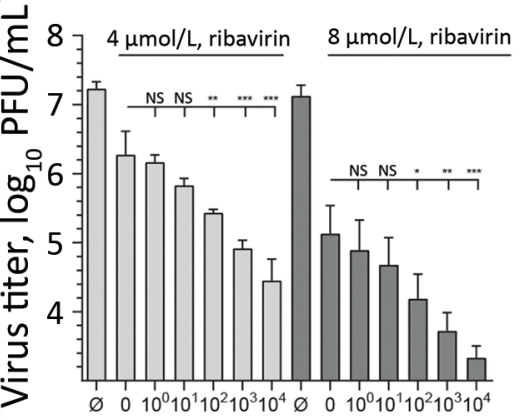

IFN $\alpha 2 \mathrm{a}, \mathrm{IU} / \mathrm{mL}$

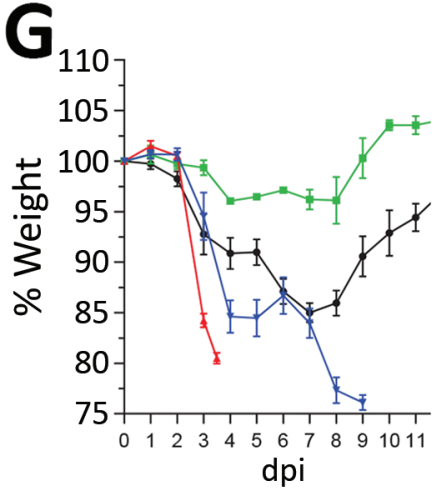

1: Untreated

2: Positive control

3: IFNa,10,000 IU/mL

4: IFN $\psi, 10,000 \mathrm{IU} / \mathrm{mL}$

5: Ribavirin, $64 \mu \mathrm{mol} / \mathrm{L}$

6: T705, $64 \mu \mathrm{mol} / \mathrm{L}$

Figure 5. Antiviral treatment against BRBV. A-D) Huh7 cells infected with BRBV (multiplicity of infection 0.001) had viral titers determined at $48 \mathrm{hpi}$. Shown are the arithmetic means ( \pm SD) of log-transformed values of 3 independent experiments. The cells were treated with increasing amounts of IFN- $\alpha 2 \mathrm{a}$, IFN- $\gamma$, or equal amounts of both IFNs $16 \mathrm{~h}$ prior and $2 \mathrm{hpi}(\mathrm{A})$; increasing amounts of the antiviral drugs favipiravir (T705) and ribavirin $2 \mathrm{hpi} \mathrm{(B,} \mathrm{C);} \mathrm{or} \mathrm{a} \mathrm{combination} \mathrm{of} \mathrm{ribavirin} \mathrm{and} \mathrm{IFN- \alpha 2a} \mathrm{(D).} \mathrm{E)} \mathrm{To} \mathrm{evaluate} \mathrm{the} \mathrm{cytotoxicity}$ of these compounds, cells were treated with the indicated concentrations for $48 \mathrm{~h}$, or as a positive control the cells were treated with lysis buffer. LDH activity in the supernatant was determined (normalized to positive control $[n=3$, mean $\pm S D]$ ). Statistical analyses were performed with a 1-way analysis of variance (Tukey multiple comparison test). F) IFNAR ${ }^{-/-}$animals $(n=5)$ treated by intraperitoneal injection with $0.9 \% \mathrm{NaCl}$ (mock-treated) or 20 or $40 \mathrm{mg} / \mathrm{kg} / \mathrm{d}$ ribavirin starting 4 hpi with 1,000 PFU of BRBV. At $4 \mathrm{dpi}$, viral titers were determined in liver, lung, spleen, and kidney. G, H) IFNAR ${ }^{-1-}$ or STAT1 ${ }^{-1-}$ mice treated until day 7 dpi with ribavirin $(40 \mathrm{mg} / \mathrm{kg} / \mathrm{d})$ as in panel F. Weight (mean \pm SEM) and survival were monitored daily. The animals were euthanized if they lost $>25 \%$ bodyweight or showed signs of severe illness. $\mathrm{H}$ ) Statistical analysis for the survival curve performed with a log-rank (Mantel-Cox) test. BRBV, Bourbon virus; dpi, days postinfection; IFN, interferon; IFNAR, type I interferon receptor; hpi, hours postinfection; KI, kidney; LI, liver; LU, lung; LDH, lactate dehydrogenase; NS, nonsignificant; SP, spleen; $\varnothing$, mock-treated (control). ${ }^{* * *} p<0.001 ;{ }^{* *} p<0.01 ;{ }^{*} p<0.05$. 
Future analysis of the seroprevalence to BRBV in humans is urgently needed to evaluate the zoonotic spread of the virus in the affected area.

\section{Acknowledgments}

We would like to thank our colleagues from the Centers for Disease Control and Prevention's Division of Vector-Borne Disease, National Center for Emerging and Zoonotic Infectious Diseases (Fort Collins, CO, USA), for providing us with a seed stock of Bourbon virus; Rick Randall (University of St. Andrews) for his constant support and for providing the transduced A549 cell lines; Sophie Hambleton (Newcastle University Medical School) for the STAT2-deficient fibroblast cultures; and Peter Staeheli for his support with the in vivo experiments. We also thank our colleagues Otto Haller, Peter Staeheli, Martin Schwemmle, and Laura Graf for their critical reviews of our manuscript.

The work was funded by Germany's National Platform on Zoonoses Research and Federal Ministry of Food and Agriculture through the Federal Office for Agriculture and Food (grant no. 2816HS008) and by the German Research Foundation (Deutsche Forschungsgemeinschaft) (grant no. KO 1579/12-1).

This work was conducted by J.F. in partial fulfillment of the requirements for a PhD degree from the Faculty of Biology of the University of Freiburg, Germany.

\section{About the Author}

Mr. Fuchs is a PhD student at the Institute of Virology at the Medical Center of the University of Freiburg, Germany. His research interests are vectorborne viral zoonoses and their interplay with the hosts' innate immune systems.

\section{References}

1. Kosoy OI, Lambert AJ, Hawkinson DJ, Pastula DM, Goldsmith CS, Hunt DC, et al. Novel Thogotovirus associated with febrile illness and death, United States, 2014. Emerg Infect Dis. 2015;21:760-4. http://dx.doi.org/10.3201/eid2105.150150

2. Charles M. Rare tick disease found in Payne County. Stillwater News Press [cited 2018 Apr 9]. https://www.stwnewspress.com/ news/rare-tick-disease-found-in-payne-county/article 14ee1140-04f2-11e5-966e-6b24d85953ee.html

3. Miller M. Bourbon virus linked to death of park official. Missourian [cited 2018 Apr 9]. http://www.emissourian.com/ local_news/county/bourbon-virus-linked-to-death-of-park-official/article 057f0f0a-bddd-5f7a-aa0d-735187e4c379.html

4. Miller $\bar{M}$. Bourbon virus - now listed as emerging illness. Missourian [cited 2018 Apr 9]. http://www.emissourian.com/ local news/county/bourbon-virus-now-listed-as-emerging-illnessnew-case-in/article_5d67a0e6-bf55-5cfb-9e6c-9b73896fafad.html

5. Savage HM, Burkhalter KL, Godsey MS Jr, Panella NA, Ashley DC, Nicholson WL, et al. Bourbon virus in field-collected ticks, Missouri, USA. Emerg Infect Dis. 2017;23:2017-22. http://dx.doi.org/10.3201/eid2312.170532

6. Savage HM, Godsey MS Jr, Panella NA, Burkhalter KL, Manford J, Trevino-Garrison IC, et al. Surveillance for tick-borne viruses near the location of a fatal human case of Bourbon virus (family Orthomyxoviridae: genus Thogotovirus) in eastern Kansas, 2015. J Med Entomol. 2018;55:701-5. http://dx.doi.org/10.1093/ jme/tjx251

7. McCauley JW, Hongo S, Kaverin NV, Kochs G, Lamb RA, Matrosovich MN, Perez DR, et al. Orthomyxoviridae. In: King AMQ, Lefkowitz E, Adams MJ, Carstens EB, eds. Virus taxonomy: ninth report of the International Committee on Taxonomy of Viruses. San Diego: Elsevier; 2012. p. 749-61.

8. Lambert AJ, Velez JO, Brault AC, Calvert AE, Bell-Sakyi L, Bosco-Lauth AM, et al. Molecular, serological and in vitro culturebased characterization of Bourbon virus, a newly described human pathogen of the genus Thogotovirus. J Clin Virol. 2015;73:127-32. http://dx.doi.org/10.1016/j.jcv.2015.10.021

9. Moore DL, Causey OR, Carey DE, Reddy S, Cooke AR, Akinkugbe FM, et al. Arthropod-borne viral infections of man in Nigeria, 1964-1970. Ann Trop Med Parasitol. 1975;69:49-64. http://dx.doi.org/10.1080/00034983.1975.11686983

10. Butenko AM, Leshchinskaia EV, Semashko IV, Donets MA, Mart'ianova LI. Dhori virus - a causative agent of human disease. 5 cases of laboratory infection [in Russian]. Vopr Virusol. 1987;32:724-9.

11. Mateo RI, Xiao SY, Lei H, DA Rosa AP, Tesh RB. Dhori virus (Orthomyxoviridae: Thogotovirus) infection in mice: a model of the pathogenesis of severe orthomyxovirus infection. Am J Trop Med Hyg. 2007;76:785-90. http://dx.doi.org/10.4269/ ajtmh.2007.76.785

12. Li G, Wang N, Guzman H, Sbrana E, Yoshikawa T, Tseng CT, et al. Dhori virus (Orthomyxoviridae: Thogotovirus) infection of mice produces a disease and cytokine response pattern similar to that of highly virulent influenza A (H5N1) virus infection in humans. Am J Trop Med Hyg. 2008;78:675-80. http://dx.doi.org/10.4269/ ajtmh.2008.78.675

13. Lanford RE, Guerra B, Lee H, Averett DR, Pfeiffer B, Chavez D, et al. Antiviral effect and virus-host interactions in response to alpha interferon, gamma interferon, poly(i)-poly(c), tumor necrosis factor alpha, and ribavirin in hepatitis $\mathrm{C}$ virus subgenomic replicons. J Virol. 2003;77:1092-104. http://dx.doi.org/10.1128/ JVI.77.2.1092-1104.2003

14. Hambleton S, Goodbourn S, Young DF, Dickinson P, Mohamad SM, Valappil M, et al. STAT2 deficiency and susceptibility to viral illness in humans. Proc Natl Acad Sci U S A. 2013;110:3053-8. http://dx.doi.org/10.1073/pnas.1220098110

15. Clay WC, Fuller FJ. Nucleotide sequence of the tick-borne orthomyxo-like Dhori/India/1313/61 virus membrane protein gene. J Gen Virol. 1992;73:2609-16. http://dx.doi.org/10.1099/ 0022-1317-73-10-2609

16. Albanese M, Bruno-Smiraglia C, Di di Cuonzo G, Lavagnino A, Srihongse S. Isolation of Thogoto virus from Rhipicephalus bursa ticks in western Sicily. Acta Virol. 1972;16:267.

17. Mordstein M, Kochs G, Dumoutier L, Renauld JC, Paludan SR, Klucher K, et al. Interferon-lambda contributes to innate immunity of mice against influenza A virus but not against hepatotropic viruses. PLoS Pathog. 2008;4:e1000151. http://dx.doi.org/10.1371/journal.ppat.1000151

18. Thimme R, Frese M, Kochs G, Haller O. Mxl but not MxA confers resistance against tick-borne Dhori virus in mice. Virology. 1995;211:296-301. http://dx.doi.org/10.1006/ viro.1995.1404

19. Mosca JD, Pitha PM. Transcriptional and posttranscriptional regulation of exogenous human beta interferon gene in simian cells defective in interferon synthesis. Mol Cell Biol. 1986;6:2279-83. http://dx.doi.org/10.1128/MCB.6.6.2279

20. Hilton L, Moganeradj K, Zhang G, Chen YH, Randall RE, McCauley JW, et al. The NPro product of bovine viral diarrhea virus inhibits DNA binding by interferon regulatory factor 3 and 
targets it for proteasomal degradation. J Virol. 2006;80:11723-32. http://dx.doi.org/10.1128/JVI.01145-06

21. Didcock L, Young DF, Goodbourn S, Randall RE. The V protein of simian virus 5 inhibits interferon signalling by targeting STAT1 for proteasome-mediated degradation. J Virol. 1999;73:9928-33.

22. Schroder K, Hertzog PJ, Ravasi T, Hume DA. Interferon-gamma: an overview of signals, mechanisms and functions. J Leukoc Biol. 2004;75:163-89. http://dx.doi.org/10.1189/jlb.0603252

23. Kochs G, Anzaghe M, Kronhart S, Wagner V, Gogesch P, Scheu S, et al. in vivo conditions enable IFNAR-independent type I interferon production by peritoneal CD11b+ cells upon Thogoto virus infection. J Virol. 2016;90:9330-7. http://dx.doi.org/10.1128/ JVI.00744-16

24. Biron CA, Brossay L. NK cells and NKT cells in innate defense against viral infections. Curr Opin Immunol. 2001;13:458-64. http://dx.doi.org/10.1016/S0952-7915(00)00241-7

25. Centers for Disease Control and Prevention. Bourbon virus [cited 2018 Apr 9]. https://www.cdc.gov/ncezid/dvbd/bourbon/index.html

26. Rowe T, Banner D, Farooqui A, Ng DC, Kelvin AA, Rubino S, et al. In vivo ribavirin activity against severe pandemic H1N1 influenza A/Mexico/4108/2009. J Gen Virol. 2010;91:2898-906. http://dx.doi.org/10.1099/vir.0.024323-0

27. Furuta Y, Gowen BB, Takahashi K, Shiraki K, Smee DF, Barnard DL. Favipiravir (T-705), a novel viral RNA polymerase inhibitor. Antiviral Res. 2013;100:446-54. http://dx.doi.org/10.1016/j.antiviral.2013.09.015

28. Tsubota A, Akuta N, Suzuki F, Suzuki Y, Someya T, Kobayashi M, et al. Viral dynamics and pharmacokinetics in combined interferon alfa-2b and ribavirin therapy for patients infected with hepatitis $\mathrm{C}$ virus of genotype $1 \mathrm{~b}$ and high pretreatment viral load. Intervirology. 2002;45:33-42. http://dx.doi.org/10.1159/000050085

29. Feld JJ, Hoofnagle JH. Mechanism of action of interferon and ribavirin in treatment of hepatitis C. Nature. 2005;436:967-72. http://dx.doi.org/10.1038/nature04082

Address for correspondence: Georg Kochs, University of Freiburg, Institute of Virology, University Medical Center, Hermann-

Herder-Strasse 11, 79104 Freiburg, Germany; email:

georg.kochs@uniklinik-freiburg.de

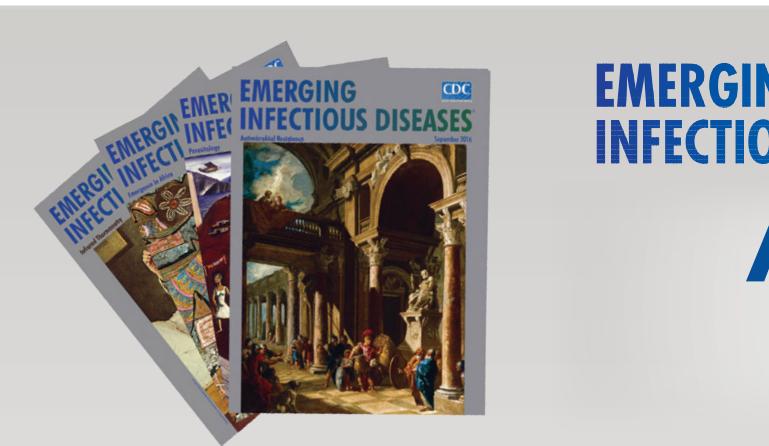

- Co-Infections in Visceral Pentastomiasis, Democratic Republic of the Congo

- Multistate US Outbreak of Rapidly Growing Mycobacterial Infections Associated with Medical Tourism to the Dominican Republic, 2013-2014

- Virulence and Evolution of West Nile Virus, Australia, 1960-2012

- Phylogeographic Evidence for 2 Genetically Distinct Zoonotic Plasmodium knowlesi Parasites, Malaysia

- Hemolysis after Oral Artemisinin Combination Therapy for Uncomplicated Plasmodium falciparum Malaria

- Enterovirus D68 Infection in Children with Acute Flaccid Myelitis, Colorado, USA, 2014

- Middle East Respiratory Syndrome Coronavirus Transmission in Extended Family, Saudi Arabia, 2014

- Exposure-Specific and Age-Specific Attack Rates for Ebola Virus Disease in Ebola-Affected Households, Sierra Leone

- Outbreak of Achromobacter xylosoxidans and Ochrobactrum anthropi Infections after Prostate Biopsies, France, 2014
- Human Babesiosis, Bolivia, 2013

- Assessment of Community Event-Based Surveillance for Ebola Virus Disease, Sierra Leone, 2015

- Probable Rabies Virus Transmission through Organ Transplantation, China, 2015

- Cutaneous Melioidosis Cluster Caused by Contaminated Wound Irrigation Fluid

- Possible Role of Fish and Frogs as Paratenic Hosts of Dracunculus medinensis, Chad

- Time Lags between Exanthematous IIIness Attributed to Zika Virus, Guillain-Barré Syndrome, and Microcephaly, Salvador, Brazil

- Use of Unamplified RNA/cDNA-Hybrid Nanopore Sequencing for Rapid Detection and Characterization of RNA Viruses

- Importation of Hybrid Human-Associated Trypanosoma cruzi Strains of Southern South American Origin, Colombia

- Lyssavirus in Indian Flying Foxes, Sri Lanka

- Survival and Growth of Orientia tsutsugamushi in Conventional Hemocultures

\section{To revisit the February 2016 issue, go to: \\ https://wwwnc.cdc.gov/eid/articles/issue/22/2/table-of-contents}

